\title{
S \\ Effect of crystalline disorder on quantum tunneling in the single-molecule magnet $\mathrm{Mn}_{12}$ benzoate
}

\author{
C. Carbonera, F. Luis, * J. Campo, J. Sánchez-Marcos, A. Camón, and J. Chaboy \\ Instituto de Ciencia de Materiales de Aragón, CSIC-Universidad de Zaragoza, 50009 Zaragoza, Spain \\ and Departamento de Física de la Materia Condensada, Universidad de Zaragoza, 50009 Zaragoza, Spain \\ D. Ruiz-Molina and I. Imaz \\ Centro de Investigación en Nanociencia y Nanotecnología CIN2 (CSIC-ICN), Campus UAB, Edificio CM7, \\ 08193 Cerdanyola del Vallés, Spain \\ J. van Slageren \\ School of Chemistry, University of Nottingham, Nottingham NG7 2RD, United Kingdom \\ and 1. Physikalisches Institut, Universität Stuttgart, Pfaffenwaldring 57, 70550 Stuttgart, Germany \\ S. Dengler \\ 1. Physikalisches Institut, Universität Stuttgart, Pfaffenwaldring 57, 70550 Stuttgart, Germany \\ M. González \\ Institut Laue Langevin, Grenoble, France
}

(Received 15 September 2009; revised manuscript received 1 December 2009; published 29 January 2010)

\begin{abstract}
We report a detailed study of the effects that crystalline disorder has on the magnetic relaxation and quantum tunneling of $\mathrm{Mn}_{12}$ benzoate clusters. Thanks to the absence of interstitial molecules in the crystal structure of this molecular compound, we have been able to isolate the influence of long-range crystalline disorder. For this, we compare results obtained under two extreme situations: a crystalline sample and a nearly amorphous material. The results show that crystalline disorder affects little the anisotropy, magnetic relaxation, and quantum tunneling of these materials. It follows that disorder is not a necessary ingredient for the existence of magnetic quantum tunneling. The results unveil, however, a subtle influence of crystallinity via the modification of the symmetry of dipole-dipole interactions. The faster tunneling rates measured for the amorphous material are accounted for by a narrower distribution of dipolar bias in this material, as compared with the crystalline sample.
\end{abstract}

DOI: 10.1103/PhysRevB.81.014427

PACS number(s): 75.45.+j, 75.50.Xx, 75.60.Jk, 75.50.Kj

\section{INTRODUCTION}

Crystals of single-molecule magnets are ordered lattices of nearly identical clusters with a large ground-state spin $S{ }^{1}$ Probably the best studied of these is $\mathrm{Mn}_{12}$, for which $S=10$. The combination of high spin and strong uniaxial magnetic anisotropy leads to slow magnetic relaxation and hysteresis at low temperatures, ${ }^{2}$ making them potential candidates for information storage at the molecular level. In addition, they show intriguing quantum behavior, like quantum tunneling between their spin-up and spin-down states, which can be turned on and off by the application of magnetic fields. ${ }^{3-5}$

The resonant character of the spin-tunneling process, reminiscent of the discrete magnetic level spectrum of these molecules, gives rise to steplike hysteresis loops and maxima of the magnetic-relaxation rates that are observed at the "crossing fields." Although a qualitative account of these phenomena can be achieved with the simplest model for the ideal isolated molecule, ${ }^{3-5}$ a quantitative description is much more complex and involves the interaction with the environment. In particular, the origin of the transverse energy terms (i.e., not commuting with the spin projection $S_{z}$ along the anisotropy axis) has been subject to debate ever since tunneling was first discovered in these molecules.

The fourfold symmetry of the molecule allows the existence of fourth-order off-diagonal terms. The combination of these terms with those arising from dipolar and hyperfine interactions can, in principle, induce reasonably high tunneling rates at any crossing field. ${ }^{6}$ In the last few years, however, extrinsic interactions, arising from disorder, have been proposed as the dominant sources of quantum tunneling in $\mathrm{Mn}_{12}$ nanomagnets. Two types of disorder have been considered: disorder arising from lattice defects, such as dislocations ${ }^{7,8}$ and disorder induced by the local orientation of interstitial molecules from the crystallization process. ${ }^{9}$ For the acetate derivative of $\mathrm{Mn}_{12}$, four acetic-acid molecules surrounding the molecular core are equally distributed between two equivalent orientations. Depending on this orientation, they establish strong hydrogen bonds to either of the two molecules in the unit cell. It has been argued that this bond can distort the local symmetry of the molecule, giving rise to a nonzero orthorhombic transverse term that is forbidden by the pure tetragonal symmetry. The latter scenario, which would lead to a discrete distribution of transverse terms in contrast with the continuous distribution expected for lattice defects, agrees best with experimental evidence obtained from magnetic-relaxation and electronic spinresonance experiments. ${ }^{10-12}$ However, recent experiments performed on a new $\mathrm{Mn}_{12}$ derivative, $\mathrm{Mn}_{12}$-tBuAc, which contains no interstitial solvent molecules show hysteresis loops that look very much like those measured for $\mathrm{Mn}_{12}$ 
acetate. ${ }^{13}$ The observed tunneling has been attributed, in this case, to the intrinsic anisotropy and interactions of the undistorted molecule. ${ }^{14}$ Clearly, this fundamental question is not settled yet.

The aim of the present work is to ascertain the nature of those interactions that induce quantum tunneling in $\mathrm{Mn}_{12}$ compounds and, in particular, to quantify the influence of crystalline disorder and defects. In order to approach this goal, we have followed a strategy combining: (a) the ability to experimentally tune the degree of disorder and (b) a quantitative comparison of the resulting relaxation rates with theoretical predictions. We have studied two extreme, and well characterized, experimental situations with very different degrees of crystallinity: a nearly perfectly crystalline sample, on the one hand, and an almost amorphous sample obtained by a fast precipitation process. We have chosen a derivative of $\mathrm{Mn}_{12}, \mathrm{Mn}_{12}$ benzoate $\left(\mathrm{Mn}_{12} \mathrm{bz}\right)$, which contains no interstitial molecules of crystallization. By eliminating the influence of these molecules, we aim to isolate the, probably weaker, effects caused only by long-range crystalline disorder. A previous study of this material addressed the influence that decreasing crystal's size has on the magnetic relaxation and tunneling. ${ }^{15}$

Another distinctive aspect of our work is the combination of diverse and complementary experimental techniques, ac susceptibility, electron-spin resonance, and neutron scattering, which provide complete information on the parameters that determine the magnetic-relaxation process: energy levels and tunneling rates. The very broad time window (spanning nearly 11 decades) covered by ac-susceptibility and magnetic-relaxation experiments enables us also to separate the contribution of the "normal" $\mathrm{Mn}_{12}$ clusters from that of the fast-relaxing species. ${ }^{16-19}$

As the text that follows shows, disorder makes $\mathrm{Mn}_{12}$ spins tunnel faster than they would do in a perfect crystal. However, this faster tunneling does not seem to be associated with a change in the anisotropy parameters that determine the energy barrier between spin-up and spin-down states and the tunneling probabilities. It is rather caused by a narrower distribution of bias dipolar fields, resulting from the different spatial organization of the molecules. Our results show also that the presence of interstitial molecules or any other source of disorder is not a necessary ingredient for the existence of quantum tunneling.

This paper is organized as follows. In Sec. II we give experimental details, including the sample preparation methods, the characterization of the degree of disorder as well as the other physical characterization techniques. The experimental results obtained by these techniques are given in Sec. III. The combination of ac susceptibility, magnetization, and spectroscopic techniques enables us to determine which of the parameters that determine the magnetization dynamics is most sensitive to a change in crystallinity. In Sec. IV, several models that might account for the influence of disorder on quantum tunneling are tested against the experimental results. Section V is left for the conclusions.

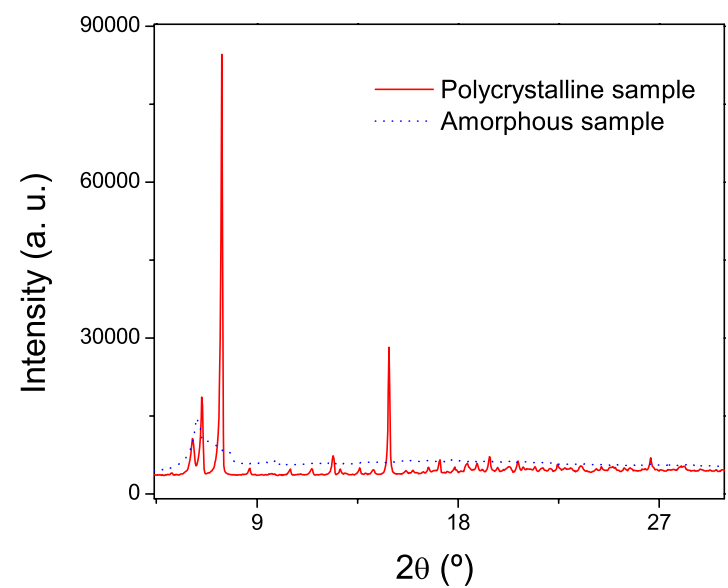

FIG. 1. (Color online) X-ray powder-diffraction patterns of the crystalline and amorphous $\mathrm{Mn}_{12} \mathrm{bz}$ samples measured at room temperature.

\section{SAMPLES AND EXPERIMENTS}

\section{A. Samples: preparation and characterization of the crystalline disorder}

The crystalline $\mathrm{Mn}_{12} \mathrm{bz}$ sample was synthesized following a previously described method. ${ }^{20}$ In order to prepare a noncrystalline sample, a fast precipitation process induced by the addition of hexane to a methylene chloride solution of the $\mathrm{Mn}_{12} \mathrm{bz}$ sample was used.

The degree of crystalline order in the two samples was studied by $\mathrm{x}$-ray diffraction experiments. The diffraction pattern was obtained on samples deposited on a monocrystalline silica substrate to avoid any overlap of the Bragg reflections of the sample with those coming from the substrate. This configuration enables decreasing the background, which is especially important in the case of the amorphous material. Results for the two samples are shown in Fig. 1. The x-ray diffraction pattern has confirmed the high quality of the crystalline sample. Diffraction patterns measured before and after the susceptibility and ESR measurements show no evidence of aging. As far as the crystal structure is concerned, the sample exhibits the same triclinic crystalline phase that was described by Sessoli et al. ${ }^{20}$

The data for the quenched sample, hereafter referred to as the "amorphous" sample, show that most of its initial crystalline character is lost. Yet, it still exhibits some crystalline character as can be seen from the low-angle region in Fig. 1, which has been detected thanks to the use of a nondisturbing substrate. This is in agreement with our preliminary experiments that showed the enormous tendency to crystallize of this compound, and therefore, the difficulties to get a pure amorphous material. From the position of the broad peaks observed, it is possible that the ordered regions have the phase described by Takeda and co-workers. ${ }^{21}$ In fact, this result is very similar to that obtained by the technique of compressed fluids, where a very fast crystallization process also leads to the same crystalline phase. ${ }^{22}$ Regarding the local order at the Mn sites, previous X-ray absorption spectroscopy experiments have shown that the local environment of the $\mathrm{Mn}$ ions is not modified by either varying the crystal's size $^{15}$ or the degree of crystallinity. ${ }^{23}$ 


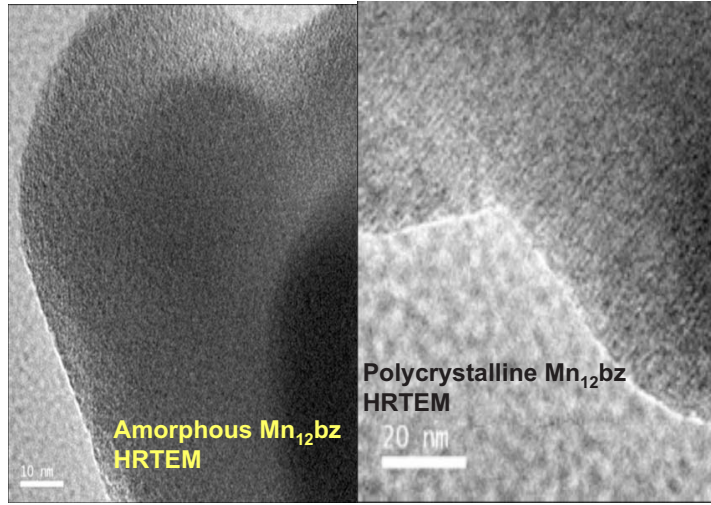

FIG. 2. (Color online) High-resolution TEM images of the amorphous (left) and crystalline (right) $\mathrm{Mn}_{12}$ bz samples.

The two samples have also been studied by highresolution transmission electron microscopy (HRTEM). The goal was to characterize the grain size and shape and to add more information about their degree of crystalline order. To avoid the local heating and degradation of the sample by the electron beam, the experiments were performed while keeping the sample under a flow of nitrogen gas evaporating from the liquid. Typical HRTEM images of the two samples are shown in Fig. 2. Although the average size of the grains is similar in both, the microstructure is easily distinguishable. In the case of the crystalline sample, it is possible to observe large areas with a neat organization in crystalline planes. For the amorphous material, the regions of local crystalline order do not exceed a lateral size of about $10 \mathrm{~nm}$. A sphere of this diameter contains approximately 160 molecules (or 20 unit cells).

\section{B. Experimental techniques}

ac-susceptibility data were measured between $0.05 \mathrm{~Hz}$ and $1.4 \mathrm{kHz}$ by means of a commercial superconducting quantum interference device (SQUID) magnetometer. Above $100 \mathrm{~Hz}$, susceptibility data were also measured with a PPMS experimental platform that uses a conventional inductive method. In both cases, the samples were mixed with grease that avoids, at low temperatures, the rotation of the powder grains by the action of external magnetic fields. All experiments were performed on powdered samples, to ensure that the overall orientation of the magnetic anisotropy axes is the same (random) in the amorphous and crystalline samples. The contributions of the grease and of the sample holder were measured separately and subtracted from the data. They were anyway negligible with respect to the magnetic responses of the samples.

Magnetic-relaxation experiments were performed using two different experimental setups, depending on the temperature region. Above $1.8 \mathrm{~K}$, we employed the high-sensitivity reciprocating sample option (RSO) of a commercial SQUID magnetometer. The samples were first cooled from $10 \mathrm{~K}$ down to the measuring temperature under an applied magnetic field of $10 \mathrm{Oe}$. Once the temperature was stabilized at its final values, the magnetic field was switched off and the ensuing decay of the magnetization was measured as a func- tion of time. Magnetic-relaxation and hysteresis experiments in the region between 0.35 and $1.5 \mathrm{~K}$ were performed with a homemade micro-Hall magnetometer installed in a ${ }^{3} \mathrm{He}$ refrigerator.

ESR spectra were measured on the two samples by using a quasioptical cw terahertz spectrometer described in literature. $^{24,25}$ This spectrometer was used for recording frequency-domain magnetic-resonance spectroscopy (FDMRS) spectra, by sweeping the radiation frequency in zero applied field, as well as high-frequency ESR (HFESR) spectra, in which case it is the magnetic field that is swept at a fixed frequency $\omega / 2 \pi=300 \mathrm{GHz}$. FDMRS spectra were simulated with the use of a home-written program ${ }^{26}$ while HFESR spectra were simulated with Weihe's simulation software. ${ }^{27}$

Inelastic neutron-scattering (INS) experiments were performed at the experimental line IN5 of the Institue Laue Langevin. The wavelength of the incoming neutrons was $\lambda$ $=5.9 \AA$ and the instrumental energy resolution was $\sigma$ $=0.029 \mathrm{meV}$. The peaks observed at positive (neutron energy loss) and negative (neutron energy gain) energy transfers were fitted to Gaussian profiles having an energy width determined by the instrumental resolution in order to extract the energy of the different neutron-induced transitions.

\section{EXPERIMENTAL RESULTS}

\section{A. ac susceptibility: slow- and fast-relaxing clusters}

ac-susceptibility data measured for frequencies $\omega / 2 \pi$ between $0.1 \mathrm{~Hz}$ and $10 \mathrm{kHz}$ are shown in Fig. 3. They show the typical superparamagnetic freezing associated with the slow relaxation of these molecules, which takes place below a blocking temperature $T_{\mathrm{b}}$ that decreases with $\omega$. A closer look to these data reveals a first important difference between the two samples. For the amorphous sample, an additional susceptibility peak shows up at temperatures below $T_{\mathrm{b}}$. Peaks like these are in fact observed for most $\mathrm{Mn}_{12}$ derivatives, and arise from the presence of fast relaxing (FR) $\mathrm{Mn}_{12}$ clusters. From these data, we conclude that FR molecules represent only $2 \%$ of all clusters in the crystalline sample, almost a rarity indicating again its good quality as reference sample. By contrast, this fraction becomes about $45(2) \%$ in the amorphous material.

Above $T_{\mathrm{b}}, \chi^{\prime}$ attains its equilibrium value $\chi_{\text {eq }}$. For a powder sample and in the limit, valid here, of strong anisotropy $\chi_{\text {eq }}$ should follow a Curie-Weiss law, ${ }^{28}$

$$
\chi_{\text {eq }}=N_{\mathrm{A}} \frac{g^{2} \mu_{\mathrm{B}}^{2} S^{2}}{3 k_{\mathrm{B}}(T-\theta)}
$$

as indeed it does for the two samples. The best fit to Eq. (1) with $S=10$ gives $g=2.01$ and 1.87 for the crystalline and amorphous samples, respectively. The different $g$ values we find for the two samples can be probably ascribed to the different concentration of FR molecules. ${ }^{18}$ The other parameter of the fit, the Weiss temperature $\theta$, depends on the strength of intermolecular interactions. We get $\theta \simeq-0.54 \mathrm{~K}$ for the polycrystalline sample and $-0.2 \mathrm{~K}$ for the amorphous one. This result suggests that the distribution in dipolar bias, 


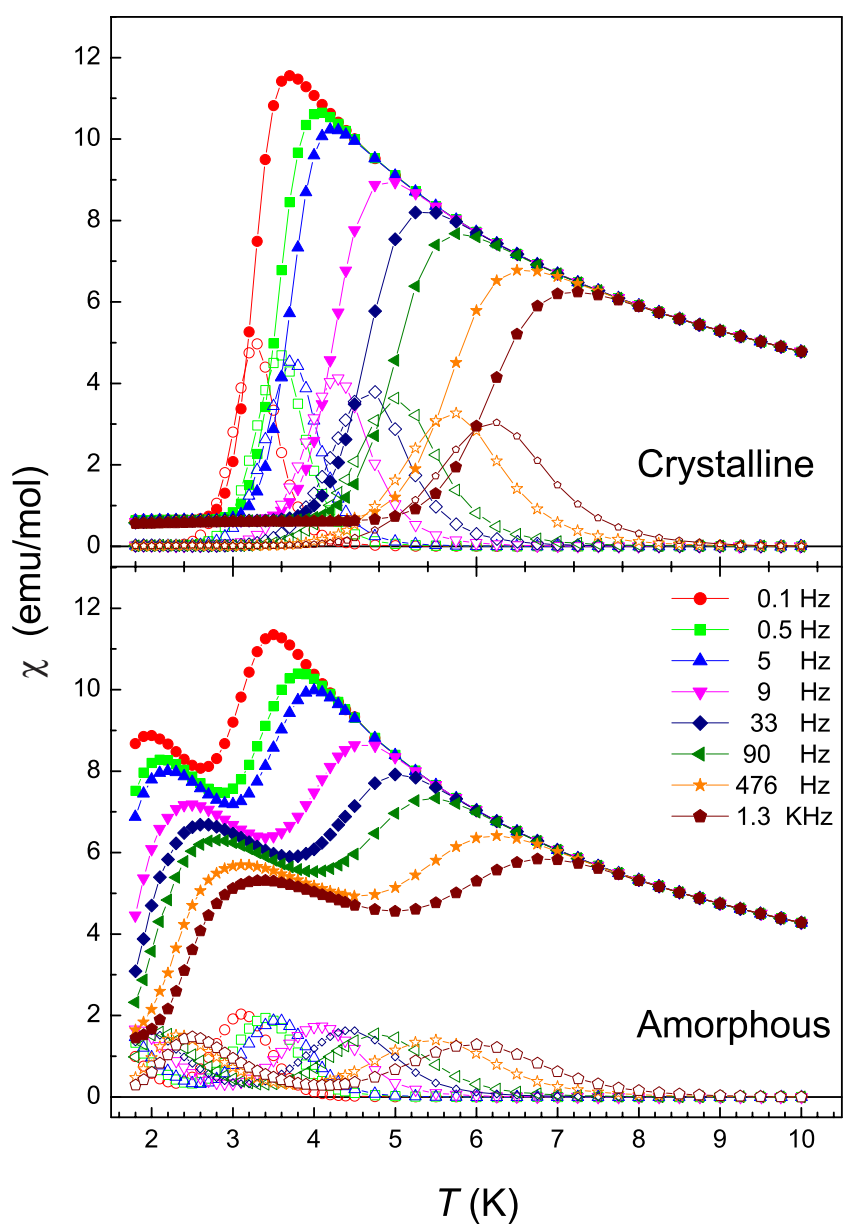

FIG. 3. (Color online) ac-susceptibility data of the polycrystalline (top panel) and the amorphous (bottom panel) samples of $\mathrm{Mn}_{12}$ bz. Solid symbols represent the in-phase susceptibility component $\chi^{\prime}$ and open symbols represent the out-of-phase $\chi^{\prime \prime}$ component.

i.e., in the component parallel to the molecular anisotropy axis, becomes therefore narrower (i.e., typical dipolar magnetic fields are weaker) in the latter sample. ${ }^{29}$

\section{B. Relaxation times at zero field}

The average relaxation time can be estimated with acceptable precision from the temperature of the imaginary susceptibility maxima and the condition that $\tau \simeq 1 / \omega$ at these temperatures. A more rigorous ${ }^{4,28,30}$ estimate of $\tau$ and its distribution can be obtained from experiments performed at fixed temperatures by changing the time scale. Since $\tau$ is strongly dependent on $T$, covering a broad region of temperatures requires a combination of different experimental methods: ac susceptibility versus frequency for sufficiently high temperatures (or equivalently for sufficiently short time scales) and time-dependent magnetic-relaxation experiments for the region of low temperatures. We again emphasize that the contributions to the susceptibility of the standard [or slow relaxing (SR)] and FR species are separated by several frequency decades and can therefore be determined independently of each other. In the case of magnetic-relaxation experiments, the long-time magnetization decay measured

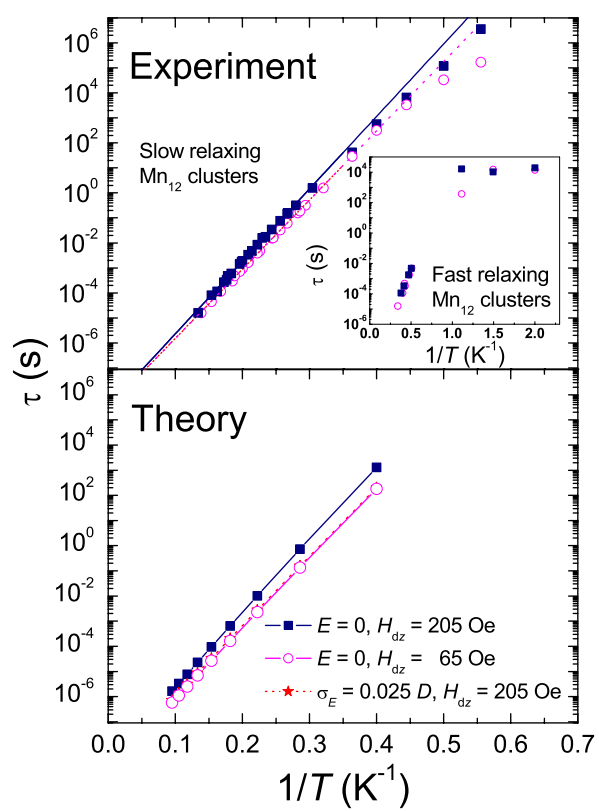

FIG. 4. (Color online) (Top panel) Relaxation time for the standard molecules of $\mathrm{Mn}_{12} \mathrm{bz}$ in the polycrystalline (squares) and amorphous (circles) samples. Solid lines are least-squares fits using Arrhenius' law, Eq. (2). The inset shows the relaxation time for the fast-relaxing molecules of $\mathrm{Mn}_{12} \mathrm{bz}$ in a polycrystalline and an amorphous sample. Solid lines are least-squares fits with Arrhenius' law, Eq. (2). (Bottom panel) Relaxation time of $\mathrm{Mn}_{12}$ calculated using the model described in Ref. 6 for three sets of parameters that represent: (a) the crystalline material, with perfect fourth-order molecular symmetry $\left(E=0\right.$ and $\left.H_{d z}=205 \mathrm{Oe}\right)$ and (b) two possible effects of disorder: a weaker average bias dipolar field $H_{d z}$ $=65 \mathrm{Oe}$ and the existence of a Gaussian distribution, with a width $\sigma_{E}=0.025 D$, of orthorhombic parameters $E$, associated with molecular distortions induced by strains (Refs. 7 and 8). The parameters used to calculate the latter two curves were chosen so as to give the same activation energy $U=63.7 \mathrm{~K}$ found for the amorphous material. These curves are, for this reason, virtually identical.

above $1.8 \mathrm{~K}$ mainly reflects the contribution of the standard clusters. By contrast, data measured below $1 \mathrm{~K}$ provide only information about the decay of the FR species. Results of these experiments are described next.

The frequency-dependent susceptibility data have been fitted, at each temperature, with a Cole-Cole function ${ }^{31}$ to extract the average magnetic-relaxation time $\tau$ and a parameter $\beta$ ( $\equiv 1-\alpha$ with $\alpha$ being the parameter defined in Ref. 31) that accounts for the distribution of relaxation times. In particular, we get $\beta \simeq 0.73$ and $\beta \simeq 0.88$ for the standard $\mathrm{Mn}_{12}$ in the amorphous and crystalline samples, respectively. The fact that $\beta<1$ shows the existence of a distribution in relaxation times, which is broader for the amorphous material. The same conclusion can be drawn from time-dependent magnetic-relaxation experiments. The long-time relaxation curves are nonexponential but can be fitted by using a stretched exponential function. ${ }^{32,33}$ We shall not consider the origin of the distribution here but leave it for the discussion.

Relaxation times obtained for the two $\mathrm{Mn}_{12}$ species are shown in Fig. 4. The relaxation times of the FR $\mathrm{Mn}_{12}$ found in the crystalline and amorphous materials are nearly identi- 
cal. They both show a crossover between a thermally activated regime at high temperatures, where $\tau$ obeys Arrhenius' law,

$$
\tau=\tau_{0} \exp \left(\frac{U}{k_{\mathrm{B}} T}\right)
$$

with $\tau_{0} \simeq 10^{-9}$ s and $U=30(1) \mathrm{K}$ to a nearly constant value. This suggests that direct tunneling via the ground state becomes the dominant relaxation mechanism of these FR molecules below approximately $0.8 \mathrm{~K}$.

In the case of SR $\mathrm{Mn}_{12}$, the differences between the two samples are also small. Yet, we observe that standard clusters relax faster in an amorphous environment at any temperature. As a first approximation, it is possible to describe the whole temperature variation in $\tau$ above $2 \mathrm{~K}$ by Arrhenius' law. We get very similar prefactors $\tau_{0}\left[\tau_{0}=5(1) \times 10^{-9} \mathrm{~s}\right.$ for the amorphous and $\tau_{0}=7(1) \times 10^{-9}$ for the crystalline] whereas $U$ of the two samples differs by only $2.5 \%[U$ $=60.8(1) \mathrm{K}$ for the amorphous and $U=62.3(5) \mathrm{K}$ for the crystalline]. However, as it is often the case with these materials, ${ }^{34}$ the activation energy depends on temperature, reflecting the competition between several relaxation processes associated with different tunneling states (see the discussion in Sec. IV). The difference in activation energies is more pronounced above $3.5 \mathrm{~K}$. In this "high-temperature" region, we get $U=66.7(1) \mathrm{K}$ and $\tau_{0}=2.9 \times 10^{-9} \mathrm{~s}$ for the crystalline and $U=63.7(1) \mathrm{K}$ and $\tau_{0}=2.6 \times 10^{-9} \mathrm{~s}$ for the amorphous, respectively. Below $3 \mathrm{~K}, \tau$ vs $1 / T$ curves tend to become approximately parallel until they diverge again near $2 \mathrm{~K}$. It is important to mention that $\tau_{0}$ measured at high temperatures provides an estimate of the lifetimes of excited magnetic states, which are determined by the spin-phonon interactions. ${ }^{6}$ Our data therefore suggest that the degree of disorder or the spatial arrangement of the clusters affects these interactions very little. ${ }^{35}$

\section{Determination of the anisotropy constants (by ESR and neutron scattering)}

The previous section leaves open the question of which effect causes the observed variation in the effective slope $U$ of Arrhenius plots obtained from relaxation measurements in zero magnetic field and at high temperatures. Since $H=0$ is a resonance field, a tempting explanation would be that the larger amount of disorder in the amorphous sample leads to larger transverse anisotropy which increases the tunneling probability. Tunneling therefore would take place via lowerlying states decreasing the effective energy barrier. However, $U$ depends not only on the tunneling rates but also on the height of the total anisotropy barrier determined by the axial anisotropy. To determine this classical $U_{c l}$, we directly measured the energy splitting of the lowest spin multiplet by means of FDMRS, high-frequency ESR, and INS methods.

Figure 5 shows HFESR spectra recorded on an unrestrained polycrystalline sample at a frequency of $\omega / 2 \pi$ $=305 \mathrm{GHz}$. Due to the torque exerted by the magnetic field, the crystals tend to align their anisotropy axes with it. The results are therefore quasisingle-crystal spectra with the field aligned along the easy axis. These spectra show between one

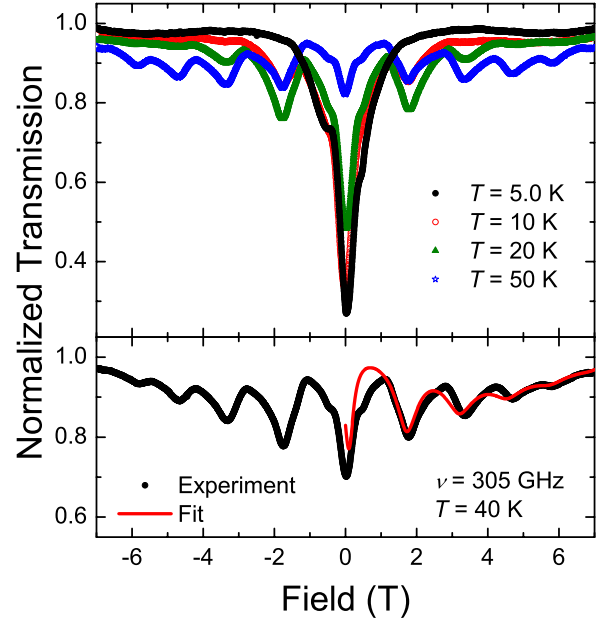

FIG. 5. (Color online) (Top panel) HFESR spectra recorded on an unrestrained sample of deuterated polycrystalline $\mathrm{Mn}_{12} \mathrm{bz}$ at $\omega / 2 \pi=305 \mathrm{GHz}$ and different temperatures as indicated in the figure. (Bottom panel) Experimental spectrum measured at $T=40 \mathrm{~K}$ and fit using the spin-Hamiltonian parameters given in the text.

(at the lowest temperature) and five (at $T=50 \mathrm{~K}$ ) clear transitions. FDMRS (not shown) and INS spectra (Fig. 6) recorded on the same polycrystalline sample show three and seven resonance lines, respectively. The resonance positions of the resonance lines observed by both methods match very well. All spectra were simulated on the basis of the following spin Hamiltonian:

$$
\mathcal{H}=-D S_{z}^{2}+A_{4} S_{z}^{4}+g \mu_{\mathrm{B}} \mathbf{S} \cdot \mathbf{H} .
$$

For the simulation of the HFESR spectra, we introduced a spread of $10^{\circ}$ in the angles that the easy axes make with the external field. The values obtained for the parameters $D$ and $A_{4}$ (Table I) are very typical for the $\mathrm{Mn}_{12}$ system and all techniques agree well on these values. The fits were not improved by the inclusion of second-order or fourth-order transverse anisotropy terms $\left[E\left(S_{x}^{2}-S_{y}^{2}\right)\right.$ and $C\left(S_{+}^{4}+S_{-}^{4}\right)$, respectively].

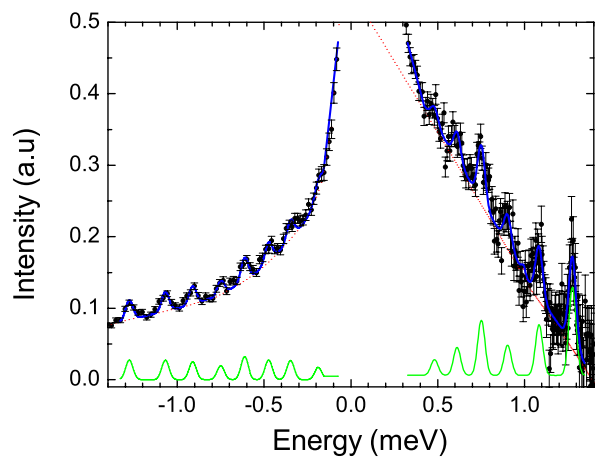

FIG. 6. (Color online) INS spectra recorded at $T=5 \mathrm{~K}$ on a sample of deuterated polycrystalline $\mathrm{Mn}_{12} \mathrm{bz}$ with $\lambda=5.9 \AA$. The thick (blue) represents the fit employed to determine the energies associated to different transitions. The fitting curve consists of a smooth background (thin red line), common to all transitions, plus a Gaussian profile (green line at the bottom) for each transition. 
TABLE I. Parameters of the Hamiltonian obtained from ESR experiments for $\mathrm{Mn}_{12} \mathrm{bz}$ crystalline and amorphous samples.

\begin{tabular}{lcccc}
\hline \hline Sample & Technique & $\begin{array}{c}D \\
(\mathrm{~K})\end{array}$ & $\begin{array}{c}A_{4} \\
(\mathrm{~K})\end{array}$ & $\begin{array}{c}U_{c l} \\
(\mathrm{~K})\end{array}$ \\
\hline $\mathrm{Mn}_{12} \mathrm{bz}$ & FDMRS & $0.58(1)$ & $-1.03(8) \times 10^{-3}$ & $68(2)$ \\
Amorph & ESR & $0.58(1)$ & $-1.01(8) \times 10^{-3}$ & $68(2)$ \\
$\mathrm{Mn}_{12} \mathrm{bz}$ & FDMRS & $0.59(1)$ & $-1.0(1) \times 10^{-3}$ & $69(2)$ \\
Policry & ESR & $0.59(2)$ & $-1.0(1) \times 10^{-3}$ & $69(3)$ \\
\hline \hline
\end{tabular}

HFESR (not shown) and FDMRS (Fig. 7) spectra were also recorded on samples of amorphous $\mathrm{Mn}_{12} \mathrm{bz}$. In the FDMRS spectra three resonance lines can be observed. Fits of the HFESR and FDMRS spectra yields parameter values (Table I) that are virtually the same as those obtained for the polycrystalline sample. On the basis of the relatively small number of resonance lines observed, no information on the possible occurrence of transverse anisotropy could be obtained. We will show below that the transverse anisotropy must be very similar for both samples. From the axial anisotropy values we can calculate the classical energy barrier $U_{c l}=D S^{2}+A_{4} S^{4}$ and we get values that are indeed very close: $U_{c l}=69.1 \mathrm{~K}$ for the crystalline sample and $U_{c l}=68.3 \mathrm{~K}$ for the amorphous material.

\section{Field-dependent susceptibility data}

Once established that the total or classical energy barriers are virtually the same, the different relaxation rates of the two materials can be attributed to different tunneling probabilities. This conclusion is supported by the results of susceptibility experiments performed at nonzero magnetic fields, as we show next. Frequency-dependent susceptibility data were measured at fixed $T=4.8 \mathrm{~K}$ and increasing mag-

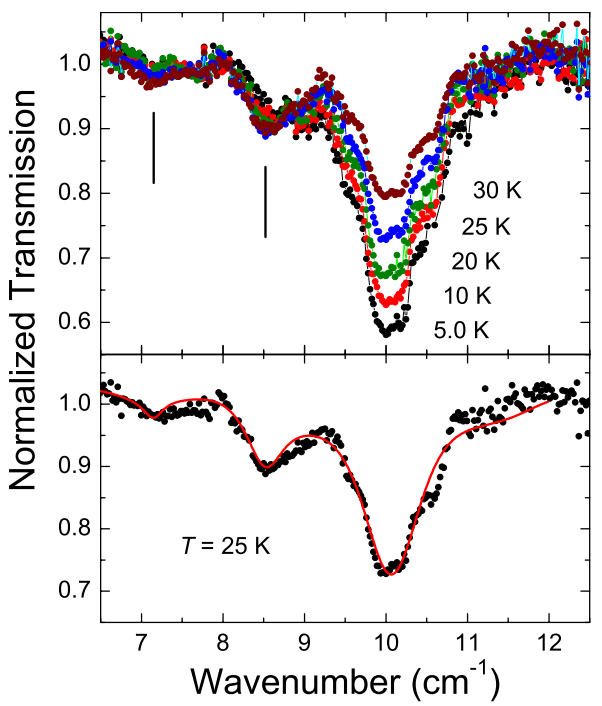

FIG. 7. (Color online) (Top panel) FDMRS spectra recorded on pressed powder pellet of amorphous $\mathrm{Mn}_{12} \mathrm{bz}$ at different temperatures as indicated in the figure. (Bottom panel) Experimental data and fit for the $25 \mathrm{~K}$ spectrum.

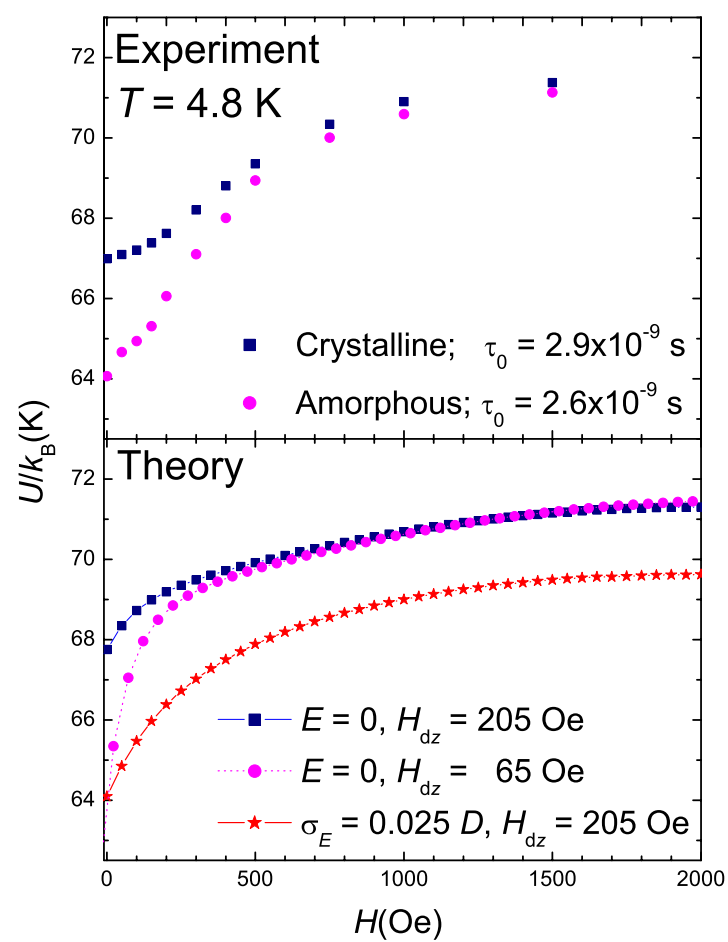

FIG. 8. (Color online) (Top panel) Average activation energy obtained from relaxation-time data for the two samples measured at $T=4.8 \mathrm{~K}$. (Bottom panel) Average activation energies obtained from calculations of the relaxation time at $T=4.8 \mathrm{~K}$. The parameters are the same as those given in the caption of Fig. 4. Notice that, in the calculations, the field was applied along the anisotropy axis $z$.

netic fields. This temperature was chosen because the maximum of $\chi^{\prime \prime}$ lies near the center of the frequency window. Since we know the prefactor $\tau_{0}$, the effective activation energy $U(H)$ can be obtained ${ }^{30}$ from $\tau(H)$ as $U=k_{\mathrm{B}} T \ln \left(\tau / \tau_{0}\right)$. Results obtained in this way are plotted in Fig. 8. As $H$ increases, $U$ increases too, as expected because a bias magnetic field breaks the degeneracy of the spin-up and spindown states between which tunneling takes place. Of crucial importance to the present study is the fact that the difference in $U$ vanishes (within experimental errors) above $800 \mathrm{Oe}$. This behavior confirms that the classical energy barrier $U_{c l}$ is the same for both the polycrystalline and the amorphous samples and provides direct evidence for the enhanced tunneling rates [i.e., smaller $U(H=0)]$ in the spatially disordered sample.

\section{DISCUSSION}

The results of the experiments described in the previous section show that the magnetic-relaxation rates of $\mathrm{Mn}_{12}$ clusters are little affected by the degree of crystalline disorder. Only small differences are observed between two extreme situations. The main conclusion is therefore that the presence of defects is not essential to account for the thermally activated quantum tunneling observed in $\mathrm{Mn}_{12}$ singlemolecule magnets. Disorder does however modify the tunneling rates, which become higher in the amorphous mate- 
rial. The goal of the present section is to discuss different mechanisms that might account for the observed changes. For this, we shall make a quantitative comparison between the results of our experiments and the predictions of several of the theoretical models that have been proposed to explain how disorder affects the quantum-tunneling process.

The relaxation rate describing quantum tunneling via a pair of nearly degenerate states $| \pm m\rangle$ has a Lorentzian shape as a function of the longitudinal bias $\xi_{m}=2 m g \mu_{\mathrm{B}} H_{z}$,

$$
\Gamma(m) \simeq 2 \Gamma_{0, m}\left[\Delta_{m}^{2} / \xi_{m}^{2}+w_{m}^{2}\right] \exp \left(-U_{m} / k_{\mathrm{B}} T\right),
$$

where $\Gamma_{0, m}$ is the probability of decaying to other levels via the absorption or emission of phonons, $\Delta_{m}$ is the tunnel splitting of the pair $| \pm m\rangle$ induced by energy terms not commuting with $S_{z}, w_{m}^{2}$ defines the width of the Lorentzian, and $U_{m}$ is the energy of the levels measured from the ground level. Explicit forms for $w_{m}$ have been found in the limits of (i) large coupling to the phonon bath $\hbar \Gamma_{0, m} \gg \Delta_{m},{ }^{36}$ where $w_{m}$ $\simeq \hbar \Gamma_{0, m}$ and (ii) weak coupling $\hbar \Gamma_{0, m} \ll \Delta_{m}$, in which $w_{m}$ $\simeq \Delta_{m}$. A simple expression that interpolates between these limits is $w_{m}^{2}=\Delta_{m}^{2}+\hbar^{2} \Gamma_{0, m}^{2} \cdot{ }^{37}$

Equation (4) can be seen as the typical Arrhenius' law weighted by the factor within brackets, which measures how much the states $\pm m$ are "linked" by tunneling. This factor results from a competition between those interactions that induce tunneling (they enhance $\Delta_{m}$ ) and those that contribute to either the bias $\xi$ or the homogenous level width $\hbar \Gamma_{0, m}$ and therefore inhibit quantum tunneling. Notice that $\xi$ is finite even if no magnetic field is applied to the sample, on account of the fields created by dipolar interactions between the molecular spins as well as by hyperfine interactions with nuclear spins.

In principle, these three parameters are all affected by disorder. In what follows, we shall try to find out which of these is the dominant source of the changes observed in $\tau$. Let us first consider spin-phonon interactions. These interactions control the lifetime of the excited magnetic states, thus also the prefactor of the Arrhenius law that holds in the limit of high temperatures. Indeed, in this limit, states near the top of the anisotropy energy barrier are populated. These states have $\Delta_{m}>\xi$ and $\Delta_{m}>\hbar \Gamma_{0, m}$. Under these conditions, $\Gamma_{m}$ $\simeq \Gamma_{0, m} \exp \left(-U_{m} / k_{\mathrm{B}} T\right)$, that is $\tau_{0} \simeq 1 / \Gamma_{0, m}$. As we have discussed already in section, the experiments suggest that $\tau_{0}$, and thus also the homogenous broadening of the levels, are nearly independent of the degree of disorder. This applies to the slow- and fast-relaxing $\mathrm{Mn}_{12}$ species.

Recent theoretical models ${ }^{7-9}$ suggest that distortions, caused by either local disorder in the position of solvent interstitial molecules or by other defects, enable the existence of terms of the spin Hamiltonian, such as $E\left(S_{x}^{2}-S_{y}^{2}\right)$, which would be forbidden in the case of a perfectly symmetric molecular core. These terms induce quantum tunneling and could, at least partly, contribute to decrease $\tau$. Yet, in contrast with the situation of $\mathrm{Mn}_{12}$ acetate, the benzoate derivative does not contain any interstitial molecule inside the crystal structure. The latter compound provides therefore a nearly ideal situation to study the sole effect of long-range strains, caused by, e.g., lattice dislocations or other defects. Notice that the sample that we call amorphous is in fact the result of a rapid crystallization process, which should therefore give rise to a high concentration of defects. Defects give rise to a spatial distribution of strains, thus also to a distribution of molecular distortions. According to the theory developed by Chudnovsky and Garanin, 7,8 this leads to a Gaussian distribution of the orthorhombic parameter $E$ centered around $E=0$, with a width $\sigma_{E}$ that depends on the concentration of lattice defects.

The almost complete absence of crystalline order modifies also the distribution of dipolar magnetic fields (obviously, hyperfine interactions are not affected). In a crystal, the bias is dominated by the field generated by "chains" of spins lying closest to the orientation of the magnetic anisotropy axis (say z). ${ }^{38}$ In an amorphous material, these chains are broken and therefore the angular distribution of dipolar fields becomes more isotropic. As a result, the local bias can be reduced at the expense of the other two components (along say $x$ and $y$ ) of the dipolar field. The different Weiss temperatures, $\theta=-0.54 \mathrm{~K}$ and $\theta=-0.2 \mathrm{~K}$, found, respectively, for the polycrystaline and amorphous samples, suggest that such changes occur. As a first approximation, ${ }^{29}$ we estimate the dipolar bias from these Weiss temperatures as follows: $\xi \equiv 2 g \mu_{\mathrm{B}} S H_{d, z} \simeq k_{\mathrm{B}}|\theta|$. We then find $H_{d, z} \simeq 205$ and 78 Oe for the crystalline and amorphous samples, respectively.

In order to decide between these two scenarios (i.e., whether strain-induced molecular distortions or the variation in the distribution of dipolar bias fields are responsible for the observed changes), we have performed numerical simulations of the relaxation rate introducing changes in (a) the strength of dipolar bias $\xi$ and (b) the distribution of $E$. For simplicity, we have used the model reported in Ref. 6 which is valid if either $\Delta_{m}$ or $\xi$ are much larger than $\hbar \Gamma_{0, m}$. In fact, these conditions are not unrealistic since $\hbar \Gamma_{0, m} \sim 1.7$ $\times 10^{-2} \mathrm{~K}$ for the SR molecules whereas the typical dipolar $\xi \sim k_{\mathrm{B}}|\theta|$. Furthermore, none of the arguments that follow are exclusive of this particular model.

In the lower panel of Fig. 4, we plot relaxation times obtained from the calculations in the temperature range between 3 and $7 \mathrm{~K}$. The rates were obtained, exactly as in the experiments, by fitting the variation in the calculated linear susceptibility with respect to frequency. We took $g=2$ and anisotropy parameters $D=0.6 k_{\mathrm{B}}$ and $A_{4}=-1.1 \times 10^{-3} k_{\mathrm{B}}$ that, being within the range allowed by spectroscopic determinations, fit best the classical activation energy extracted from field-dependent experiments (Fig. 8). We also included the off-diagonal term, $C\left(S_{+}^{4}+S_{-}^{4}\right)$, allowed by the fourfold symmetry of the molecular core, with $C=3 \times 10^{-5} \mathrm{~K} .{ }^{39}$ As argued above, we take $H_{d, z} \simeq 205$ Oe for the crystalline sample, which in fact gives $U=67.3 \mathrm{~K}$, as experimentally observed. We also took $H_{d, x}=H_{d, z}$, although the results calculated with this model are very weakly dependent on the actual magnitude of the transverse dipolar fields. ${ }^{40}$ As it is shown in Fig. 4, the lower $U$ observed in the amorphous material can be accounted for by either decreasing $H_{d, z}$ to 65 Oe (very close to the value of 78 Oe estimated from the Weiss temperature), with $E=0$, or by introducing a distribution of $E$ values. In the latter case, a good fit was achieved for $\sigma_{E} \simeq 0.015$, i.e., about $2.5 \%$ of the diagonal parameter $D$.

It is clear that relaxation times measured at $H=0$ do not help to establish the dominant role played by crystalline dis- 


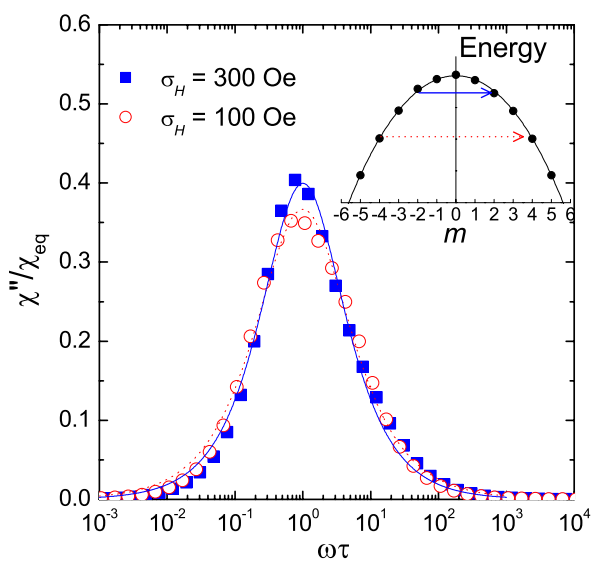

FIG. 9. (Color online) Imaginary component of the ac susceptibility, normalized by the equilibrium (i.e., zero-frequency) susceptibility and calculated for two different widths $\sigma_{H}$ of the distribution of dipolar bias. The lines are fits made using a Cole-Cole function, as it is done experimentally. They give $\beta=0.78$ and $\beta=0.86$ for $\sigma_{H}=100$ and $\sigma_{H}=300 \mathrm{Oe}$, respectively, indicating that the effective distribution of relaxation times becomes narrower when the bias distribution broadens. The inset shows a magnification of the region near the top of the magnetic energy barrier, where the two competing relaxation paths are indicated by arrows. For $\sigma_{H}=100$ Oe the two give comparable contributions to the susceptibility whereas for $\sigma_{H}=300$ Oe mainly the tunneling via $m= \pm 2$ matters.

order. For this, we need additional information that, as we argue next, can be provided by the experiments performed under applied magnetic fields. The lower panel of Fig. 8 shows the variation in the activation energy with $H_{z}$ that follows from the same model in the three situations considered before. As it might be anticipated, differences in the strength of dipolar interactions become irrelevant when the external $H_{z}$ is much larger than $H_{d, z}$. The reason is that, for large bias $\xi \gg \Delta_{m}$, tunneling is "blocked" for all levels and the classical behavior is recovered. Since in the two curves considered (for $H_{d, z}=205$ and $65 \mathrm{Oe}$ ) $U_{c l}$ is the same, the two merge in the classical limit, in good agreement with experimental results. By contrast, the activation energy calculated for a distribution of the parameter $E$ remains below the curve corresponding to $E=0$ even for large $H_{z}$. Again, this result is easy to understand and it is independent of the model. For a classical Hamiltonian $\mathcal{H}=-D S_{z}^{2}+A_{4} S_{z}^{4}-E\left(S_{x}^{2}-S_{y}^{2}\right)$ the energy barrier is, at zero field, $U_{c l}=(D-|E|) S^{2}+\left|A_{4}\right| S^{4}$. Therefore, a small orthorhombic distortion would reduce the classical barrier by about $2 \mathrm{~K}$, an effect that we do not observe experimentally. Such a reduction has, however, been observed in microparticles and nanoparticles of the same material, prepared by an ultrafast crystallization process. ${ }^{15}$ In the latter case, the very strong strain induced during the crystal growth gives probably rise to a relatively large orthorhombic distortion.

There remains a final point that deserves consideration. From the analysis of frequency-dependent susceptibility data, it follows that the effective distribution of relaxation times is broader in the case of the amorphous sample. Is this broadening compatible with a narrowing of the distribution of dipolar bias? Curiously, this counterintuitive result can also be accounted for by the numerical simulations, as shown in Fig. 9, and explained with the simple arguments that follow. For a relatively narrow distribution of bias (with say a width $\sigma_{H}=100 \mathrm{Oe}$ as in one of the examples shown), the susceptibility results from contributions, of comparable magnitude, arising from two different relaxation "paths," associated with tunneling via two different pairs of states ( $m$ $= \pm 4$ and $m= \pm 2$ in the simulations shown). These paths are schematically indicated by arrows in the energy-level scheme shown in the inset of Fig. 9. By increasing $\sigma_{H}$ to 300 Oe, the number of molecules that are able to tunnel via the lower-lying states with $m= \pm 4$ decreases so much that the relaxation, and therefore also the susceptibility, becomes dominated by the those molecules tunneling via $m= \pm 2$. The broadening of the dipolar bias distribution leads, in this particular situation, to a slower average magnetic relaxation but, paradoxically as it seems, with a smaller dispersion in the relaxation times.

\section{CONCLUSIONS}

We have investigated how crystalline disorder affects the anisotropy and magnetization dynamics of $\mathrm{Mn}_{12}$ singlemolecule magnets. For this, we have exhaustively studied two extreme situations: a pure crystalline sample and a sample with very short-range crystalline domains. The compound, $\mathrm{Mn}_{12} \mathrm{bz}$, has been chosen because it does not contain interstitial molecules in the structure.

For the standard species of $\mathrm{Mn}_{12}$, the dependencies of the relaxation rates on temperature and magnetic field are qualitatively the same. In particular, quantum tunneling is observed in both, the crystalline and amorphous situations, and it is very little affected by crystallinity. Similar qualitative conclusions have been drawn from studies of $\mathrm{Mn}_{12}$ clusters in frozen solutions. ${ }^{41}$ This shows that disorder, induced either by interstitial molecules or by other defects, is not a necessary ingredient for quantum tunneling to occur. As our numerical simulations show, reasonably high relaxation rates, comparable to those observed experimentally, can be obtained by a combination of the intrinsic anisotropy terms allowed by the undistorted molecular symmetry and terms arising from dipole-dipole interactions. This, of course, does not mean that disorder has no influence on tunneling. Its effects might become dominant under situations that involve strong distortions of the molecular core, as it happens when the clusters are deposited onto solid substrates ${ }^{42}$ or if the crystallization process is quenched very abruptly, for instance, when nanoparticles are made by the compressed fluid technique. $^{15}$

Thanks, in fact, to the absence of stronger effects, our results enabled us to unveil a rather subtle influence of disorder that arises via the modification of dipolar interactions between molecular spins. A direct comparison between the experiments and results of numerical calculations, enable us to conclude that the enhanced tunneling probabilities observed in the amorphous sample are probably caused by a narrower distribution of dipolar bias in this sample rather than by the molecular distortions associated with strains and lattice defects. 


\section{ACKNOWLEDGMENTS}

The authors benefitted from enlightening discussions with J. F. Fernández, N. Domingo, and S. Hill. Technical assistance from the Scientific Services unit of the University of Barcelona for the x-ray diffraction and HRTEM experiments is also acknowledged. This work has been partly funded by Grants No. MAT2009-13977-C03, No. MAT2008-06542-
C04, and No. CSD2007-00010 from the Spanish Ministerio de Ciencia e Innovación, and NABISUP from DGA. We acknowledge funding from Acción Integrada under Grant No. HA2006-0051 and the Network of Excellence MAGMANet. J.v.S and S.D. acknowledge the financial support of the Deutsche Forschungsgemeinschaft (DFG) and the DAAD. Ch.C. and I.I. acknowledge the Spanish Ministerio de Ciencia e Innovación.
*Corresponding author; fluis@unizar.es

${ }^{1}$ D. Gatteschi, R. Sessoli, and J. Villain, Molecular Nanomagnets (Oxford University Press, Oxford, 2006).

${ }^{2}$ R. Sessoli, D. Gatteschi, A. Caneschi, and M. A. Novak, Nature (London) 365, 141 (1993).

${ }^{3}$ J. R. Friedman, M. P. Sarachik, J. Tejada, and R. Ziolo, Phys. Rev. Lett. 76, 3830 (1996).

${ }^{4}$ J. M. Hernández, X. X. Zhang, F. Luis, J. Bartolomé, J. Tejada, and R. Ziolo, Europhys. Lett. 35, 301 (1996).

${ }^{5}$ L. Thomas, F. Lionti, R. Ballou, D. Gatteschi, R. Sessoli, and B. Barbara, Nature (London) 383, 145 (1996).

${ }^{6}$ F. Luis, J. Bartolomé, and J. F. Fernández, Phys. Rev. B 57, 505 (1998).

${ }^{7}$ E. M. Chudnovsky and D. A. Garanin, Phys. Rev. Lett. 87, 187203 (2001).

${ }^{8}$ D. A. Garanin and E. M. Chudnovsky, Phys. Rev. B 65, 094423 (2002).

${ }^{9}$ A. Cornia, R. Sessoli, L. Sorace, D. Gatteschi, A. L. Barra, and C. Daiguebonne, Phys. Rev. Lett. 89, 257201 (2002).

${ }^{10}$ S. Hill, R. S. Edwards, S. I. Jones, N. S. Dalal, and J. M. North, Phys. Rev. Lett. 90, 217204 (2003).

${ }^{11}$ E. del Barco, A. D. Kent, E. M. Rumberger, D. N. Hendrickson, and G. Christou, Phys. Rev. Lett. 91, 047203 (2003).

${ }^{12}$ S. Takahashi, R. S. Edwards, J. M. North, S. Hill, and N. S. Dalal, Phys. Rev. B 70, 094429 (2004).

${ }^{13}$ W. Wernsdorfer, M. Murugesu, and G. Christou, Phys. Rev. Lett. 96, 057208 (2006).

${ }^{14}$ A. L. Barra, A. Caneschi, A. Cornia, D. Gatteschi, L. Gorini, L. Ph. Heiniger, R. Sessoli, and L. Sorace, J. Am. Chem. Soc. 129, 10754 (2007).

${ }^{15}$ N. Domingo, F. Luis, M. Nakano, M. Muntó, J. Gómez, J. Chaboy, N. Ventosa, J. Campo, J. Veciana, and D. Ruiz-Molina, Phys. Rev. B 79, 214404 (2009).

${ }^{16}$ S. M. J. Aubin, Z. Sun, I. Guzei, A. Rheingold, G. Christou, and D. N. Hendrickson, Chem. Commun. (Cambridge) 1997, 2239.

${ }^{17}$ Z. Sun, D. Ruiz, E. Rumberger, Ch. D. Incarvito, K. Folting, A. L. Rheingold, G. Christou, and D. N. Hendrickson, Inorg. Chem. 37, 4758 (1998).

${ }^{18}$ K. Takeda, K. Awaga, T. Inabe, A. Yamaguchi, H. Ishimoto, T. Tomita, H. Mitamura, T. Goto, N. Mori, and H. Nojiri, Phys. Rev. B 65, 094424 (2002).

${ }^{19}$ M. Soler, W. Wernsdorfer, Z. M. Sun, J. C. Huffman, D. N. Hendrickson, and G. Christou, Chem. Commun. (Cambridge) 2003, 2672.

${ }^{20}$ R. Sessoli, H.-L. Tsai, A. R. Schake, Sh. Wang, J. B. Vincent, K. Folting, D. Gatteschi, G. Christou, and D. N. Hendrickson, J. Am. Chem. Soc. 115, 1804 (1993).
${ }^{21}$ K. Takeda, K. Awaga, and T. Inabe, Phys. Rev. B 57, R11062 (1998).

${ }^{22}$ M. Muntó, J. Gómez-Segura, J. Campo, M. Nakano, N. Ventosa, D. Ruiz-Molina, and J. Veciana, J. Mater. Chem. 16, 2612 (2006).

${ }^{23}$ J. Chaboy (unpublished).

${ }^{24}$ G. Kozlov and A. Volkov, Top. Appl. Phys. 74, 51 (1994).

${ }^{25}$ J. van Slageren, S. Vongtragool, B. Gorshunov, A. Mukhin, N. Karl, J. Krzystek, J. Telser, A. Müller, C. Sangregorio, and D. Gatteschi, Phys. Chem. Chem. Phys. 5, 3837 (2003).

${ }^{26}$ N. Kirchner, J. van Slageren, and M. Dressel, Inorg. Chim. Acta 360, 3813 (2007).

${ }^{27}$ C. Jacobsen, E. Pedersen, J. Villadsen, and H. Weihe, Inorg. Chem. 32, 1216 (1993).

${ }^{28}$ R. López-Ruiz, F. Luis, V. González, Angel Millán, and José Luis García-Palacios, Phys. Rev. B 72, 224433 (2005).

${ }^{29}$ In a mean-field approximation, the susceptibility of a powdered sample is given approximately by Eq. (1), with $\theta=(\lambda+4 \pi / 3$ $-N) M_{\mathrm{s}} g \mu_{\mathrm{B}} S / k_{\mathrm{B}}$. Here, $M_{\mathrm{s}}$ is the saturation magnetization per unit of volume and $N$ is the demagnetizing factor of each grain of powder (Ref. 43). The effective mean-field parameter $\lambda$ arises from a discrete sum over dipolar couplings with spins located inside the local sphere of a Lorentz construction while $4 \pi / 3$ gives the contribution of the interaction with the rest, taken as a continuum. Considering nearly spherical grains is a reasonably good approximation in the case of very fine powdered samples. Then $\theta \simeq \lambda M_{\mathrm{s}} g \mu_{\mathrm{B}} S / k_{\mathrm{B}}$. Dipolar couplings between pairs of molecular spins can favor either ferromagnetic or antiferromagnetic alignments of the spins, depending on the relative angle between the anisotropy axes and the relative position vector. For this reason, the sign of $\theta$ depends on the crystal's symmetry. It might be negative, as in the $\mathrm{Mn}_{12} \mathrm{bz}$ compound studied here, or positive, as in $\mathrm{Mn}_{12}$ acetate (Refs. 28 and 44). The approximation employed in the calculations reported here amounts to setting the typical dipolar bias seen by a spin in a macroscopically demagnetized sample as equal to one half of the effective mean field $\lambda M_{\mathrm{s}}$ in a magnetically saturated sample.

${ }^{30}$ F. Luis, J. Bartolomé, J. F. Fernández, J. Tejada, J. M. Hernández, X. X. Zhang, and R. Ziolo, Phys. Rev. B 55, 11448 (1997).

${ }^{31}$ K. S. Cole and R. H. Cole, J. Chem. Phys. 9, 341 (1941).

${ }^{32}$ C. Sangregorio, T. Ohm, C. Paulsen, R. Sessoli, and D. Gatteschi, Phys. Rev. Lett. 78, 4645 (1997).

${ }^{33}$ L. Thomas, A. Caneschi, and B. Barbara, Phys. Rev. Lett. 83, 2398 (1999).

${ }^{34}$ C. Lampropoulos, S. O. Hill, and G. Christou, ChemPhysChem 10, 2397 (2009).

${ }^{35}$ The difference in the prefactors found for the two samples amounts to a difference of just $2 \%$ in their respective speeds of sound. 
${ }^{36}$ D. A. Garanin and E. M. Chudnovsky, Phys. Rev. B 56, 11102 (1997).

${ }^{37}$ I. Tupitsyn and B. Barbara, in Magnetism: Molecules to Materials, edited by J. S. Miller and M. Drillon (Wiley-VCH, Weinheim, 2002), Vol. III, pp. 109-168.

${ }^{38}$ J. F. Fernández and J. J. Alonso, Phys. Rev. B 62, 53 (2000); J. F. Fernández, ibid. 66, 064423 (2002).

${ }^{39}$ A.-L. Barra, D. Gatteschi, and R. Sessoli, Phys. Rev. B 56, 8192 (1997); I. Mirebeau, M. Hennion, H. Casalta, H. Andres, H. U. Güdel, A. V. Irodova, and A. Caneschi, Phys. Rev. Lett. 83, 628 (1999); R. Bircher, G. Chaboussant, A. Sieber, H. U. Güdel, and H. Mutka, Phys. Rev. B 70, 212413 (2004).

${ }^{40}$ As shown recently by Henderson et al. (Ref. 45), the influence of transverse dipolar magnetic fields on the tunneling rates can be enhanced when excited states, associated to total $S$ values different than 10, are coupled with the states of the ground multiplet. This effect cannot be treated within the "giant spin" model employed here. It might contribute to enhance further the magnetic relaxation of the amorphous material, which has a nearly isotropic distribution of dipolar fields, with respect to that of the crystal.

${ }^{41}$ F. El Hallak, J. van Slageren, J. Gómez-Segura, D. Ruiz-Molina, and M. Dressel, Phys. Rev. B 75, 104403 (2007).

${ }^{42}$ M. Mannini, F. Pineider, Ph. Sainctavit, L. Joly, A. FraileRodriguez, M.-A. Arrio, Ch. Cartier dit Moulin, W. Wernsdorfer, A. Cornia, D. Gatteschi, and R. Sessoli, Adv. Mater. (Weinheim, Ger.) 21, 167 (2009).

${ }^{43}$ See, e.g., the appendix in A. Morello, F. L. Mettes, O. N. Bakharev, H. B. Brom, L. J. de Jongh, F. Luis, J. F. Fernández, and G. Aromí, Phys. Rev. B 73, 134406 (2006).

${ }^{44}$ F. Luis, J. Campo, J. Gómez, G. J. McIntyre, J. Luzón, and D. Ruiz-Molina, Phys. Rev. Lett. 95, 227202 (2005).

${ }^{45}$ J. J. Henderson, C. Koo, P. L. Feng, E. del Barco, S. Hill, I. S. Tupitsyn, P. C. E. Stamp, and D. N. Hendrickson, Phys. Rev. Lett. 103, 017202 (2009). 